\title{
L’avenir démographique du Québec et de ses régions en relation avec le vieillissement de la population
}

\author{
Jacques Légaré et Samuel Vézina ${ }^{1}$ \\ Université de Montréal
}

$\int$ a population du Québec, tout comme celle de la majorité des nations industrialisées, est engagée dans un processus de vieillissement démographique. Les naissances moins nombreuses, l'espérance de vie en hausse, sans compter l'arrivée des baby-boomers à l'âge de la retraite au cours de la prochaine décennie, tout cela modifiera certainement la structure démographique du Québec, laissant les personnes âgées prendre une place de plus en plus importante au sein de la population. La distribution géographique ainsi que la dynamique démographique particulière des Québécois sur le territoire nous amène à nous questionner sur l'effet que le phénomène du vieillissement pourrait avoir sur les différentes régions.

D'abord, bien qu'on sache que le vieillissement démographique est un phénomène qui affecte l'ensemble des sociétés occidentales, tout porte à croire que le Québec sera davantage affecté par ce processus qui est sans précédent dans l'histoire de l'humanité. De fait, les indicateurs statistiques placent le Québec (comme dans le cas d'autres phénomènes démographiques) proche de plusieurs pays européens et en marge de ses voisins que sont le reste du Canada et les États-Unis. La société québécoise s'apprête à vivre une accélération du vieillissement de sa population sur une période beaucoup plus courte qu'ailleurs. Plus exactement, d'ici les 50 prochaines années, on s'attend à ce que non seulement le nombre de personnes âgées de 65 ans et plus passe d'environ un million à plus de deux millions de personnes, mais que la proportion de ce groupe d'âge s'accroisse d'approximativement $130 \%$ pour constituer, au milieu du siècle présent, un peu moins du tiers de la population du Québec (figure 1).

Il faut bien comprendre qu'il est primordial de ne pas transposer les résultats d'analyse de la population québécoise dans son ensemble aux différentes régions de la province. Il est important effectivement d'être prudents face aux généralisations trop rapides de cette image globale qui ne traduit pas du tout les réalités régionales. En fait, au Québec, la population peut être découpée dans deux groupes bien distincts : la population de la grande région métropolitaine de Montréal principalement, à laquelle on peut ajouter celle des autres grands centres urbains québécois, et le reste, qu'on appellera ici «Québec hors RMR » (Régions métropolitaines de recensement). Ces deux groupes constituent deux ensembles numériquement semblables mais, de façon plus importante, ils sont affectés par des tendances démographiques très différentes. On réalise par le fait même la présence d'une forte polarisation spatiale de la population du Québec puisque environ un Québécois sur deux habite dans une des six grandes RMR de la province que sont Montréal principalement, mais aussi Québec, Trois-Rivières, Gatineau, Sherbrooke et Saguenay.

Par ailleurs, la concentration démographique se révèle une tendance encore plus prononcée lorsqu'on s'aperçoit qu'elle se confirme également à l'échelle régionale. En effet, la même situation affecte la plupart des autres régions métropolitaines du Québec qui, aux dépens des régions rurales, attirent, à des degrés différents d'efficacité, les flux démographiques. Dans un contexte où la croissance démographique de la pro- 
vince en entier deviendra très bientôt nulle, on comprend rapidement que, dans les régions dites «éloignées ", ou plutôt partout sur le territoire québécois exempté de ses six parcelles "métropolitaines » les plus importantes (Québec hors RMR), le déclin de la population semble inévitable et est parfois même souvent déjà enclenché (figure 2).

Conséquemment, on se doute fort bien que l'attraction des grands centres, couplée à la non-attirance des régions éloignées, s'amplifiera, ce qui exacerbera certainement les disparités régionales en matière de structure démographique, de vigueur économique et autre. À ce propos, il faut bien comprendre que la distribution de la population par groupe d'âge ne constitue pas un paramètre (une cause) du déclin démographique. Toutefois, elle n'en demeure pas moins un élément important à étudier pour évaluer l'impact de la démographie des régions sur leur économie. La meilleure preuve de cela est la population active. De fait, il apparaîtra évident que plus la part de population en âge de travailler est importante au sein d'une région, plus celle-ci sera susceptible d'être dynamique sur le plan économique puisqu'elle possèdera un effectif élevé de main-d'œuvre pouvant faire rouler l'économie. Lorsque, comme dans les pays industrialisés, la fécondité est basse et que l'espérance de vie tend toujours à augmenter, on assiste au vieillissement démographique des sociétés. Conséquemment, le vieillissement de la population peut conduire à une réduction de la main-d'œuvre disponible. Et dans le contexte actuel où les ressources humaines jouent un rôle de plus en plus important en matière de développement économique au sein de l'économie du savoir des sociétés industrialisés, il devient d'une importance capitale de regarder l'ampleur du phénomène de vieillissement démographique dans les régions éloignées comparativement aux régions plus centrales.

\section{Figure 1 - Évolution de la population du Québec pour certains groupes d'âge, 1951-2051}

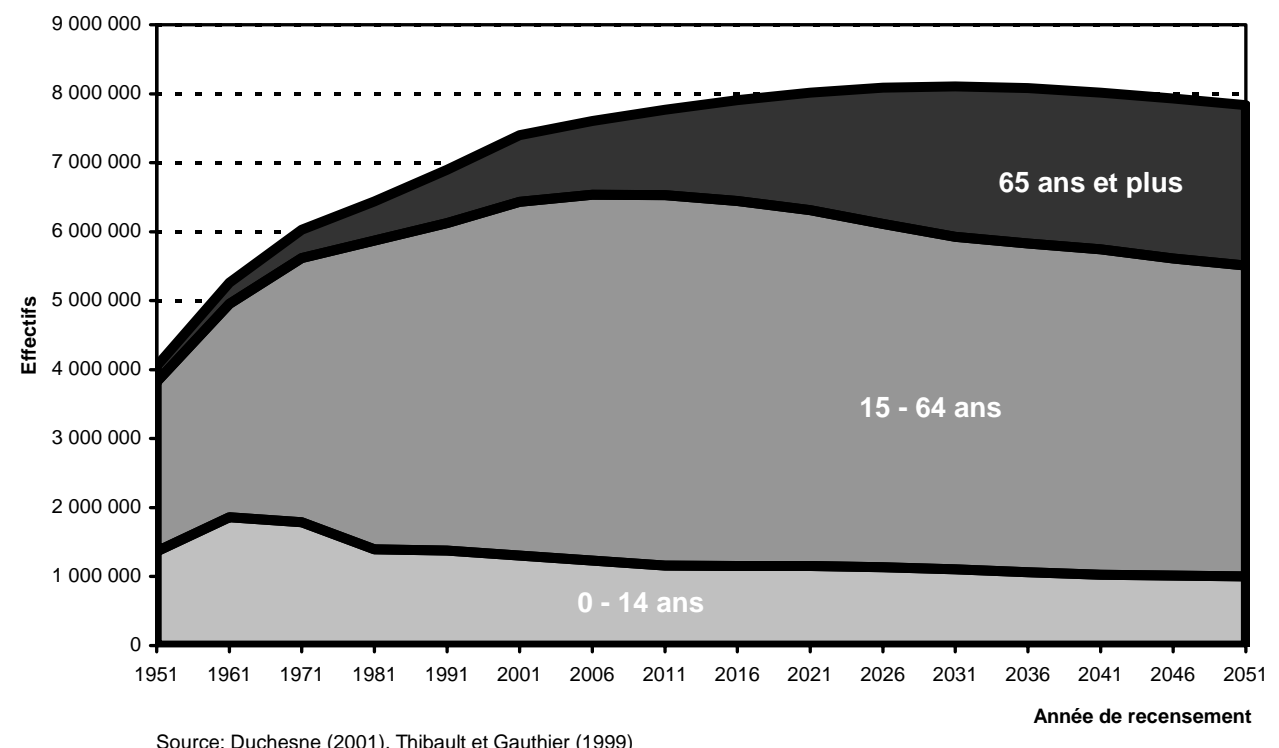

À cet effet, les perspectives démographiques prévoient que, dans ce "Québec hors RMR », la part relative des 20-64 ans passera, entre 2001 et 2026, de $61,5 \%$ à 53,5\%, constituant une baisse de $8 \%$. Cette diminution relative des effectifs d'individus en âge de travailler se traduit évidemment par une augmentation des deux groupes d'âge dépendants, soit les jeunes et les personnes âgées. Ainsi, au cours des deux prochaines décennies, le Québec (hors RMR) comprendra relativement plus de personnes âgées que la moyenne québécoise, soit près de $30 \%$ comparativement à $25 \%$ en 2026 , et $1 \%$ de moins de jeunes $(18 \%$ en régions contre $19 \%$ au Québec). Par ailleurs, des régions telles que la Gaspésie-Îles-de-la-Madeleine pourraient même être fortement touchées par le phénomène du vieillissement démographique par la combinaison des effets de deux éléments. D'abord, ces régions compteront sur leur territoire un nombre relatif de personnes âgées plus élevé que la moyenne provinciale, c'est-à-dire des proportions pouvant dé- 
passer les $35 \%$. Mais également, la part relative des jeunes âgés entre 0 et 19 ans sera vraisemblablement moindre que dans les régions métropolitaines, oscillant davantage sous la barre des $15 \%$ qu'aux alentours de $20 \%$, proportion attendue pour Montréal en 2026. Quant à l'ensemble des régions métropolitaines du Québec, on réalise rapidement que leur structure démographique sera beaucoup plus jeune et que la baisse de la proportion des actifs sera moindre, soit de
$63,7 \%$ en 2001 pour atteindre 57,8\% 25 ans plus tard. Concernant les personnes âgées, on prévoit qu'en 2026, ils compteront pour $22,6 \%$, c'est-à-dire $6 \%$ de moins que dans les régions. En somme, on $\mathrm{s}$ 'attend clairement à ce que le phénomène du vieillissement démographique affecte plus sévèrement les régions éloignées que les régions les plus urbanisées de la province.

\section{Figure 2 - Évolution relative de la population du Québec, de ses RMR et d'une de ses régions administratives, 2001-2026}

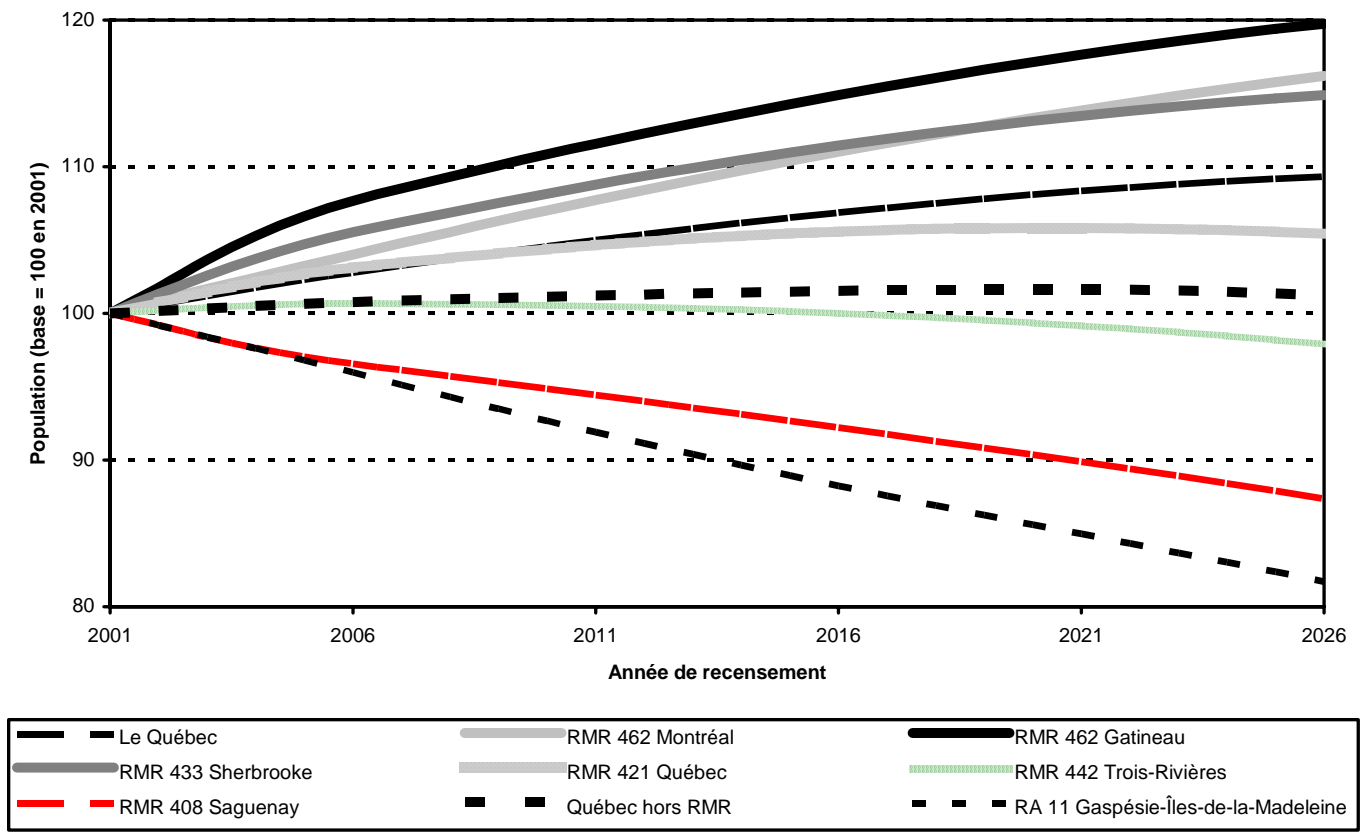

Source : Institut de la statistique du Québec, 2004.

À l'instar des tendances à la baisse de la fécondité et de la mortalité des Québécois, la composante migratoire des régions ne vient pas ralentir le processus de vieillissement démographique. D'ailleurs, comme pour bien d'autres comportements démographiques, les deux sous-populations qui forment la population du Québec démontrent des tendances diamétralement opposées. Effectivement, lorsqu'on analyse plus attentivement l'augmentation en effectifs des régions métropolitaines, on réalise très rapidement que la composante migratoire y exerce un effet considérable dont les régions dites "plus éloignées » ne bénéficient pas. En effet, en projetant la population de l'ensemble des RMR tout en faisant l'hypothèse d'une absence de migration jusqu'en 2026, on observe pratiquement une stagnation des effectifs sur les 25 années en question (figure 3). C'est exactement la situation qui est attendue pour la population québécoise des régions «hors RMR », mais de façon plus prononcée. Cela est évidemment compréhensible dans la mesure où l'on sait que la croissance de la population du Québec est stimulée par des effets de structures et aussi par des effets de comportements. Puisque les niveaux de fécondité des Québécois, qu'ils soient urbains ou ruraux (en moyenne 1,4 enfant par femme), se situent bien loin en deçà du seuil de remplacement des générations, soit 2,1 enfants par femme, un solde migratoire positif constitue le comportement démographique à adopter dans l'optique d'un accroissement de la population.

En matière de migrations internationales et interprovinciales, le Québec possède actuellement un solde positif se chiffrant aux alentours de 30000 individus. 
L'évolution de ce solde migratoire depuis les quatre dernières décennies s'est généralement tenue sous le niveau actuel et a même été longtemps négative. Quoi qu'il soit, en 2003 les entrées et les sorties vers d'autres provinces canadiennes se sont équivalues et les émigrations internationales ont été relativement peu nombreuses. On comprend dès lors que l'immigration internationale soit la composante qui ait rendu le solde migratoire positif ${ }^{2}$.

Néanmoins, la province québécoise n'attire pas et n'a jamais attiré la quote-part des immigrants qui viennent au Canada en fonction du poids démographique qu'elle occupe. En effet, quand le poids démographique du Québec dans le Canada était de l'ordre de $25 \%$, on recevait $20 \%$ des immigrants; maintenant qu'il oscille aux alentours de $20 \%$, on en reçoit environ $15 \%$. D'autre part, le Québec retient moins facilement, comparativement au reste du Canada, les gens qu'il a réussi à attirer. Par exemple, parmi les quelques 290000 immigrants qui sont entrés au Québec entre 1961 et 1971, il n'en reste sur le territoire en 2001 qu'environ $35 \%$, les autres ayant quitté le Québec (ou étant décédés). Pour les immigrants (plus d'un million) qui se sont installés dans le reste du Canada au cours de la même période, le taux de rétention est d'environ $57 \%{ }^{3}$. En somme, le Québec attire peu d'immigrants internationaux, et ceux qu'il attire sont, en définitive, rarement séduits de façon durable sur son territoire. Il demeure néanmoins pertinent d'analyser le cas de ces gens qui décident de s'installer et de vivre à long terme en sol québécois.

\section{Figure 3 - Population projetée du Québec, de ses régions métropolitaines et du reste du Québec selon deux scénarios, 2001-2026}
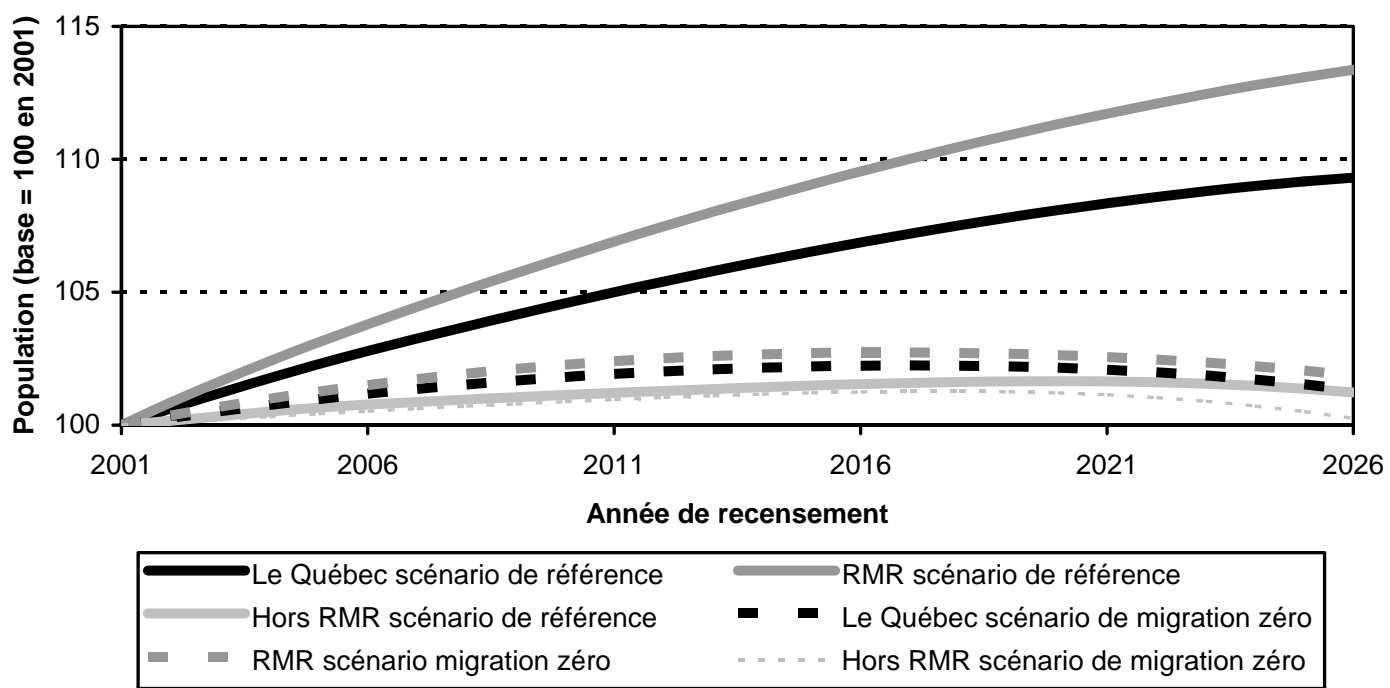

Source : Institut de la statistique du Québec, 2004

Le portrait migratoire québécois ainsi que le rôle de premier plan que joue Montréal au niveau provincial sont des éléments bien connus. Grosso modo, la grande majorité de ces nouveaux arrivants qui optent pour la province québécoise s'installent dans la région métropolitaine de Montréal. Le reste se dirige vers les autres RMR du Québec, mais celles-ci restent somme toute bien peu attractives. Il est donc rarissime de voir des immigrants aller s'installer ailleurs que dans les Régions métropolitaines de recensement. En fait, la situation migratoire des régions du Québec est frappée par l'émigration, certes vers les autres provinces canadiennes, mais davantage vers d'autres endroits au Québec, et comme de raison, principalement vers les grandes villes. Et une fois qu'on intègre les trois différents types de mouvements migratoires, on comprend mieux cette dynamique généralement attractive des grandes villes du Québec (tableau 1). 


\section{Tableau 1 - Portrait récent de la mobilité dans les régions métropolitaines de recensement du Québec, 2002-2003}

\begin{tabular}{|c|c|c|c|c|c|}
\hline \multirow[b]{2}{*}{ RMR } & \multirow[b]{2}{*}{ Entrées } & \multirow[b]{2}{*}{ Sorties } & \multirow[b]{2}{*}{ Solde } & \multicolumn{2}{|c|}{ Taux net pour 100000 habitants } \\
\hline & & & & 2002-2003 & 2001-2002 \\
\hline Saguenay & 4035 & 5354 & -1319 & $-8,4$ & $-9,2$ \\
\hline Québec & 20767 & 17807 & 2960 & 4,2 & 5,0 \\
\hline Sherbrooke & 7976 & 6621 & 1355 & 8,5 & 7,7 \\
\hline Trois-Rivières & 5639 & 5227 & 412 & 3,0 & 0,5 \\
\hline Montréal & 90079 & 68917 & 21162 & 6,0 & 7,6 \\
\hline Ottawa-Gatineau & 45926 & 38302 & 7624 & 6,8 & 8,4 \\
\hline
\end{tabular}

Source : Statistique Canada, données de l'enquête des estimations annuelles de migration par division de recensement et région métropolitaine de recensement (4101).

En s'attardant aux mouvements interrégionaux (ou infraprovinciaux, c'est-à-dire entre les régions administratives du Québec), on réalise qu'ils concernent un bassin de population beaucoup plus considérable, soit de l'ordre de 217000 personnes entre 2002 et 2003 et ce, pour ne pas parler des 500000 personnes qui déménagent chaque année sur l'ensemble du terri- toire québécois (figure 4). Cette mobilité extraordinaire, dont on parle peu en définitive, doit nécessairement être considérée sérieusement par les aménagistes du territoire québécois. Finalement, on constate que tout ce mouvement effectué par les Québécois vient alourdir cette tendance à la polarisation de la population dans les grandes agglomérations de la province.

\section{Figure 4 - Migrations internationales, interprovinciales} et infraprovinciales, Québec, 1986-2001

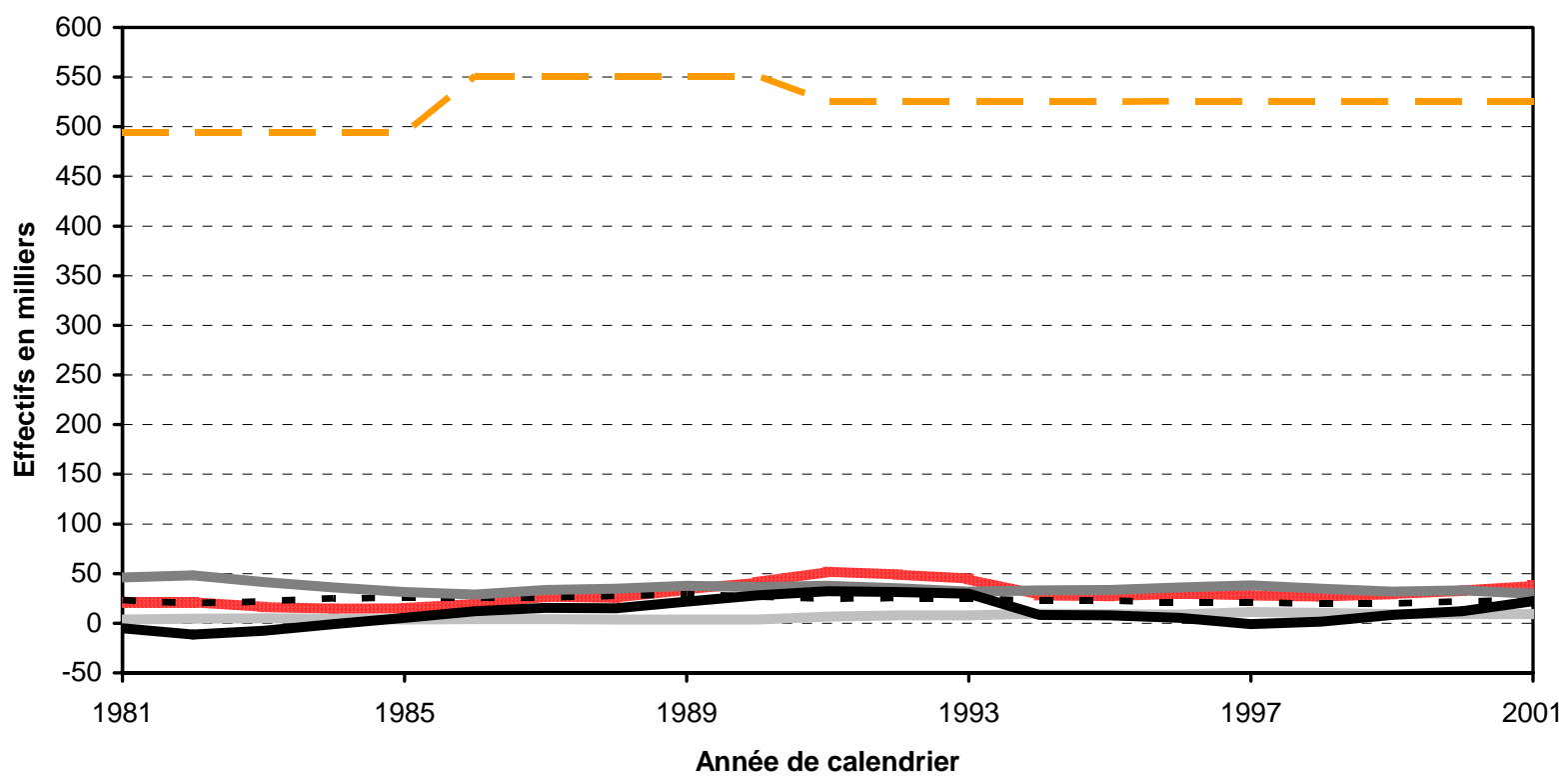

\begin{tabular}{|ll|}
\hline - - - Entrées & Émigrations \\
\hline & Total des soldes international et interprovincial \\
\hline
\end{tabular}

Source: Institut de la statistique du Québec, 26 mars 2004 et Statistique Canada (données extraites des recensements de 1986, 1991, 1996, 2001). 
Bien que la situation des régions périphériques soit difficile, celles-ci vont continuer d'exister. Il faudra toujours considérer sérieusement le rôle prépondérant de la démographie des régions et son influence fondamentale sur leur économie. Avec le temps, un nouvel équilibre devrait s'établir entre emploi et démographie. Les politiques publiques devront toujours être conçues en ce sens pour que les régions regagnent de l'attractivité et qu'éventuellement l'innovation et l'entrepreneurship émergent du tissu local et que leur économie permette d'intégrer davantage de savoir et de contenu technologique.

\section{Notes et références}

1 Jacques Légaré est professeur émérite au Département de démographie de l'Université de Montréal. Samuel Vézina est étudiant gradué au Département de démographie de l’Université de Montréal

2 Légaré, J. (2004). « Les fondements démographiques de la main-d'œuvre québécoise de demain », Gestion, Revue internationale de gestion, septembre, p. 17.

3 Ibidem. 